\title{
MURSKII'S ALGEBRA DOES NOT SATISFY MIN
}

\author{
Sheila OATES-WILLIAMS
}

This note shows that the variety generated by Murskii's 3-element algebra contains an infinite descending chain of subvarieties, thus falsifying a conjecture made previously by the author and M.R. Vaughan-Lee.

In a recent paper [1] by myself and Vaughan-Lee, the variety $M$ generated by Murskii's algebra $M$, [2] was put forwarded as a candidate for an example of a variety generated by a finite algebra which satisfies the descending chain condition on subvarieties, but not the ascending chain condition, and it was proved therein that this variety has an infinite ascending chain of subvarieties. The purpose of this note is to prove that it also has an infinite descending chain of subvarieties, so we must look elsewhere for the sort of example we were seeking in [1].

The main tool used is the theory of Shallon algebras, introduced by Shallon in her PhD thesis [3].

DEFINITION. Let $\Gamma$ be a graph (directed or undirected) with vertices $a_{1}, \ldots, a_{n}$. Then the Shallon algebra $A(\Gamma)$ has as elements $\left\{0, a_{1}, \ldots, a_{n}\right\}$ with a single binary operation defined by $a_{i} a_{j}=a_{i}$ if there is an edge from $a_{i}$ to $a_{j}$ and all other products being zero (as usual, an undirected edge is treated as two directed edges in opposite directions).

Thus, for example, Murskii's algebra itself, which has multiplication

Received 18 February 1980. 
table

\begin{tabular}{l|lll} 
& 0 & 1 & 2 \\
\hline 0 & 0 & 0 & 0 \\
1 & 0 & 0 & 1 \\
2 & 0 & 2 & 2
\end{tabular}

corresponds to the graph of Figure I:

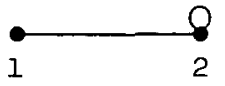

Figure 1

Clearly, any algebra with a zero and a single binary operation in which each product is either zero or equal to the first factor determines a graph of which it is the Shallon algebra; in particular, subalgebras and quotient algebras of Shallon algebras are again Shallon algebras. Of course, the direct product of two Shallon algebras will not, except in the most trivial cases, be itself a Shallon algebra. However, we do have the following result:

LEMMA. Let $A_{1}, \ldots, A_{n}$ be ShaZzon algebras obtained from the graphs $\Gamma_{1}, \ldots, \Gamma_{n}$, then the relation $\rho$ on $A_{1} \times \ldots \times A_{n}$ defined by $\left(a_{1}, \ldots, a_{n}\right) \rho\left(b_{1}, \ldots, b_{n}\right)$ if either

$$
\text { there exists } i, j \text { such that } a_{i}=b_{j}=0
$$

or

$$
a_{i}=b_{i} \quad(i=1, \ldots, n)
$$

is a congruence relation and $\left(A_{1} \times \ldots \times A_{n}\right) / \rho$ is a Shallon algebra corresponding to the graph $\Gamma_{1} \times \ldots \times \Gamma_{n}$ (P.J. Cameron is responsible for the last remark).

Proof. That $\rho$ is a congruence is immediate. Also the product of two $n$-tuples $\left(a_{1}, \ldots, a_{n}\right)$ and $\left(b_{1}, \ldots, b_{n}\right)$ with no nonzero entries will itself have no non-zero entries precisely when $a_{i} b_{i}=a_{i}$ for all $i$, that is, precisely when there is an edge from vertex $\left(a_{1}, \ldots, a_{n}\right)$ to 
vertex $\left(b_{1}, \ldots, b_{n}\right)$ in the direct product $\Gamma_{1} \times \ldots \times \Gamma_{n}$.

COROLLARY. If $A\left(\Gamma_{1}\right), \ldots, A\left(\Gamma_{n}\right)$ belong to a variety $\mathrm{V}$ so does $A\left(\Gamma_{1} \times \ldots \times \Gamma_{n}\right)$.

(Note, however, that it is only for induced subgraphs that we can say that if $\Delta$ is a subgraph of $\Gamma$ then $A(\Delta)$ is a subalgebra of $A(\Gamma)$ and so belongs to $\operatorname{var}(A(\Gamma))$.)

In [3], it is shown that $\underline{\underline{M}}$ contains every Shallon algebra arising from a loopless undirected graph. As [3] is not so readily available, I shall give a sketch of the proof here.

THEOREM 1. Let $\Gamma$ be a loopless undirected graph, then $A(\Gamma)$ is isomorphic to a quotient of a subalgebra of a cartesian product of copies of $M$.

Proof. Let $S$ denote the set of vertices of $\Gamma$. For each vertex $\gamma$ of $\Gamma$ we define a function $\phi_{\gamma}: S \times S \rightarrow M$ as follows:

$$
\begin{aligned}
& \phi_{\gamma}(\alpha, \beta)=2 \text { if } \alpha \neq \gamma \neq \beta, \\
& \phi_{\gamma}(\alpha, \gamma)=\phi_{\gamma}(\gamma, \alpha)= \begin{cases}2 \text { if } \gamma \text { is adjacent to } \alpha \text { in } \gamma, \\
y \text { otherwise. }\end{cases}
\end{aligned}
$$

If we take the subalgebra of $M^{S \times S}$ generated by the $\phi_{\gamma}$ and factor out by the congruence $\rho$ of the lemma, we have an algebra isomorphic to $A(\Gamma)$.

(A similar, though simpler construction, was used in [1], 4.1, to show that $\left.A\left(K_{n}\right) \in \underline{\underline{M}}.\right)$

The following theorem gives the promised example of an infinitely descending chain of subvarieties in $\underline{\underline{M}}$.

THEOREM 2. Let $\underline{\mathrm{U}}_{n}=\operatorname{var}\left(A\left(C_{2_{n+1}}\right)\right), n \geq 2$, then $\underline{\mathrm{U}}_{n}>\stackrel{\mathrm{U}}{n+1}_{n+1}$.

Proof. $A\left(c_{2 n+1}\right)=\left\{0, a_{1}, \ldots, a_{2 n+1} \mid a_{i} a_{i+1}=a_{i}\right\}$ (where the subscripts are taken modulo $2 n+1$ and all other products are zero). Consider 


$$
\begin{aligned}
&\left\{(0,0),\left(a_{1}, a_{1}\right),\left(a_{2}, a_{2}\right), \ldots,\left(a_{2 n}, a_{2 n}\right),\left(a_{2 n+1}, a_{2 n-1}\right),\right. \\
&\left.\left(a_{1}, a_{2 n}\right),\left(a_{2}, a_{2 n+1}\right)\right\} \subseteq A\left(c_{2 n+1}\right) \times A\left(c_{2 n+1}\right) .
\end{aligned}
$$

Modulo the congruence $\rho$ of the lemma, this clearly gives an algebra isomorphic to $A\left(C_{2 n+3}\right)$, so $\underline{\underline{U}}_{n+1} \leq \underline{\mathrm{U}}_{n}$. To show the containment is strict, it is sufficient to exhibit a law which holds in $A\left(C_{2 n+3}\right)$, but not in $A\left(C_{2 n+1}\right)$, such a law is $x_{1}\left(x_{2}\left(x_{3}\left(\ldots\left(x_{2 n}\left(x_{2 n+1} x_{1}\right)\right) \ldots\right)\right)\right)=0$. The substitution $x_{i}=a_{i}$ of elements of $A\left(C_{2 n+1}\right)$ gives the left hand

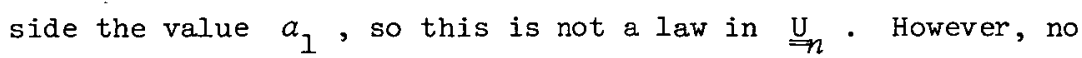
substitution of elements of $A\left(C_{2 n+3}\right)$ can give all pairs of adjacent $x_{i}$ 's in the expression on the left hand side values which are adjacent in $c_{2 n+3}$, so this is a law in $\underline{\mathrm{U}}_{n+1}$.

(Note that, although the above construction of $A\left(C_{2 n+3}\right)$ from $A\left(C_{2 n+1}\right)$ fails for $n=1$, it is possible to derive $A\left(C_{5}\right)$ from $A\left(C_{3}\right)$ so the above chain really starts at $\underline{\underline{U}}_{1}$. A similar construction yields an infinite descending chain from the cycles of even length, though in this case we really do have to start at $A\left(C_{6}\right)$, as $A\left(C_{4}\right)$ satisfies $x_{1}\left(x_{2}\left(x_{3} x_{4}\right)\right)=x_{1}\left(x_{4}\left(x_{3} x_{2}\right)\right)$ which is not satisfied by $\left.A\left(c_{6}\right).\right)$

The theory of Shallon algebras appears to be a particularly useful tool in the study of $\underline{\underline{M}}$, and perhaps could be used to decide the question as to whether or not $\underline{\underline{M}}$ contains uncountably many subvarieties.

\section{References}

[1] Sheila Oates Macdonald and M.R. Vaughan-Lee, "Varieties that make one Cross", J. Austral. Math. Soc. Ser. A 26 (1978), 368-382.

[2] V.L. MurskiY, "The existence in three-valued logic of a closed class with finite basis, not having a finite complete system of identities", Soviet Math. Dokl. 6 (1965), 1020-1024. 
[3] Carol ine Ruth Shallon, "Non-finitely based binary algebras derived from lattices" ( $\mathrm{PhD}$ thesis, University of California, Los Angeles, 1979).

Department of Mathematics,

University of Queensland,

St Lucia,

Queensland 4067,

Australia. 